\section{Dysphagia Caused by Plummer-Vinson Syndrome}

Among the various types of dysphagia, cervical dysphagia is one of the rarer ones, although differences exist over the actual incidence of esophageal webs in relation to symptoms of dysphagia [1]. PlummerVinson syndrome comprises the triad of cervical dysphagia, iron-deficiency anemia, and postcricoid esophageal web. Dysphagia and even webs are relatively common occurrences in isolation, but the complete triad of the Plummer-Vinson syndrome is a rare occurrence $[1,2]$.

A 45-year-old Saudi lady presented with 4 years of dysphagia with regard to solid foods, and a mild weight loss. Physical examination revealed angular stomatitis, glossitis, and koilonychia (Figure 1). Initial blood investigations revealed the following: hemoglobin $6.9 \mathrm{~g} / \mathrm{dl}$, mean corpuscular hemoglobin 18pg, mean corpuscular volume $60 \mathrm{fl}$; platelets $329 \times 10^{9} / 1$; leucocytes $4.8 \times 10^{9} / 1$; serum iron $5 \mu \mathrm{mol} / 1$ (14$32 \mu \mathrm{mol} / 1)$; ferritin $1 \mu \mathrm{g} / 1 \quad(8-140 \mu \mathrm{g} / \mathrm{l})$. The patient then underwent esophagogastroduodenoscopy, which revealed a longitudinal whitish web extending like a curtain across the entire diameter of the esophagus near the upper esophageal sphincter (Figure 2). Subsequently, the web was disrupted endoscopically using the biopsy forceps. Following the endoscopy session, the patient's symptoms resolved completely.

Most of the reports included in previous studies do not display the clinical triad essential for the diagnosis of PlummerVinson syndrome [1-3]. This disease occurs mostly in middle-aged women [4]. The pathogenesis of the syndrome is not well understood, although underlying iron deficiency is being touted as the possible underlying explanation [4]. The fact that this disease is extremely rare in certain regions where iron deficiency is endemic casts doubt on this interesting theory [3]. The incidence of iron deficiency is relatively high in the Saudi population, yet to our knowledge, this is the first case report of this disease from our region. While cineradiography is a sensitive method of investigating these patients, nevertheless endoscopy is a safe and reliable means of diagnosing upper esophageal webs [5]. In addition to establishing the diagnosis, treatment can be offered in the same endoscopic setting. The patient was commenced on iron therapy with substantial improvement of her symptoms and the

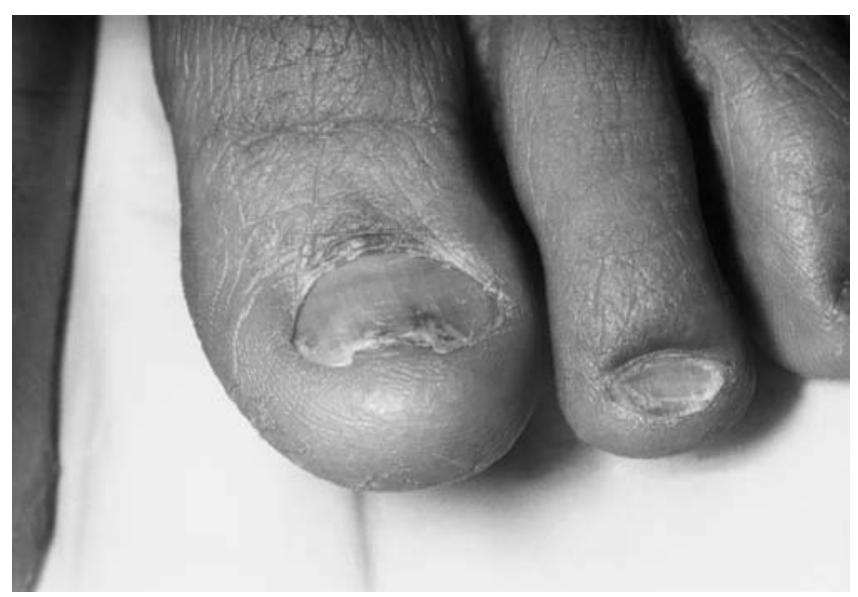

Figure 1 The patient had koilonychia

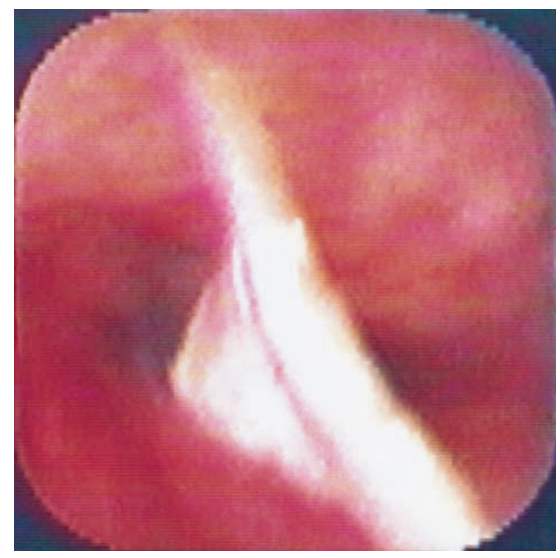

Figure 2 An endoscopic image of the esophageal web

blood count. Repeat endoscopy showed normal mucosa and no evidence of recurrence.

\section{F. M. Sanai, A. E. Mohamed,}

M. A. Al Karawi

Dept. of Gastroenterology,

Armed Forces Hospital, Riyadh,

Saudi Arabia

\section{References}

${ }^{1}$ Ekberg O, Nylander G. Webs and weblike formations in the pharynx and cervical esophagus. Diagn Imaging 1983; 52: $10-18$

${ }^{2}$ Hoffman RM, Jaffe PE. Plummer-Vinson syndrome. A case report and literature review. Arch Intern Med 1995; 155: 2008-2011

${ }^{3}$ Elwood PC, Jacobs A, Pitman RG, Entwistle CC. Epidemiology of the Patterson-Kelly syndrome. Lancet 1964; 2: 716-720

${ }^{4}$ Shamma'a MH, Benedict EB. Esophageal webs: a report of 58 cases and an attempt at classification. N Engl J Med 1958; 259: 378-384

${ }^{5}$ Low DE, Hill LD. Cervical esophageal web associated with Zenker's diverticulum. Am J Surg 1988; 156: 34-37

Corresponding Author

M. A. Al Karawi, M.D.

Armed Forces Hospital (A41)

PO Box 7897

Riyadh 11159

Saudi Arabia

Fax: $\quad+00-966-1-4791000 \times 4802$ or 4764132

E-mail: makarawi@yahoo.com 